\title{
Updates on Vaccine Safety and Post-Licensure Surveillance for Adverse Events Following Immunization in South Korea, 2005-2017
}

\author{
Dongwon Yoon, Ju Hwan Kim, Hyesung Lee, and Ju-Young Shin \\ School of Pharmacy, Sungkyunkwan University, Suwon, Korea.
}

\begin{abstract}
Purpose: Vaccine hesitancy is among the top ten threats to global health, and access to precise data on adverse events following immunization (AEFIs) is imperative to alleviate public concerns surrounding vaccines. This study aimed to present the overall trends of AEFIs reported in South Korea.

Materials and Methods: We evaluated the trends of AEFIs using the Korea Institute of Drug Safety \& Risk Management-Korea Adverse Event Reporting System database between January 2005 and December 2017. AEFIs were classified into five categories to evaluate associations between vaccines and AEFIs through a case-non-case study: neurologic reactions, general systemic reactions, local reactions, allergic reactions, and others.

Results: Among 54378 reported adverse events (AEs) associated with all vaccines approved in South Korea, more than half (56.7\%) occurred following influenza vaccination, followed by the pneumococcal (11.6\%) and Bacillus Calmette-Guérin (BCG) vaccines (5.0\%). After immunization with most vaccines, general systemic reactions were most common, followed by local and neurologic reactions. Adjusted reporting odds ratios were calculated for all neurologic, general, local, and allergic reactions: of all vaccines, rotavirus [neurologic 2.43, 95\% confidence interval (CI), 2.25-2.62], BCG (general; 2.20, 95\% CI, 1.91-2.53), BCG (local; $3.15,95 \%$ CI, 2.69-3.68), and Japanese encephalitis (allergic 2.38, 95\% CI, 1.98-2.87) vaccines showed the highest values.

Conclusion: The majority of reported AEFIs were non-serious and mostly general systemic reactions. Sufficient knowledge on the AEFIs would secure public confidence on the safety of vaccines, thereby reducing public health burden from vaccine-preventable diseases.
\end{abstract}

Key Words: Vaccines, product surveillance, postmarketing, drug-related side effects and adverse reactions, adverse drug reaction reporting systems, signal detection

\section{INTRODUCTION}

Vaccination is one of the most effective methods of priming a host's immune system against infectious diseases, especially in children and older adults who are vulnerable to infections.

\footnotetext{
Received: December 20, 2019 Revised: April 10, 2020

Accepted: May 21, 2020

Corresponding author: Ju-Young Shin, PhD, School of Pharmacy, Sungkyunkwan University, 2066 Seobu-ro, Jangan-gu, Suwon 16419, Korea.

Tel: 82-31-290-7702, Fax: 82-31-292-8800, E-mail: shin.jy@skku.edu

-The authors have no potential conflicts of interest to disclose

(c) Copyright: Yonsei University College of Medicine 2020

This is an Open Access article distributed under the terms of the Creative Commons Attribution Non-Commercial License (https://creativecommons.org/licenses/ by-nc/4.0) which permits unrestricted non-commercial use, distribution, and reproduction in any medium, provided the original work is properly cited.
}

From a public health perspective, vaccination has contributed to not only significant reduction in disease burden but also to developing herd immunity, which can protect even unvaccinated individuals. Although vaccination is perceived as a remarkable public health measure, vaccine hesitancy was listed by the World Health Organization (WHO) as one of the top ten threats to global health in 2019. ${ }^{1}$ Some individuals tend to be reluctant to undergo immunization because of adverse events following immunization (AEFIs). ${ }^{2}$ Hence, a precise assessment of AEFIs and continuous safety surveillance are important to alleviate public concerns surrounding vaccination.

Continuous surveillance for AEFIs and research on the causal relationship between vaccines and adverse events (AEs) have been undertaken globally. In the U.S, vaccine safety surveillance for all U.S. licensed vaccines was conducted using the Vaccine 
Adverse Events Reporting System from 1990. ${ }^{3}$ Australia has been publishing annual surveillance reports on AEFIs since $2004{ }^{4}$ Likewise, Switzerland ${ }^{5}$ and Brazil have also conducted AEFI surveillance studies. ${ }^{6}$

Meanwhile, although immunization during pregnancy is important to prevent morbidity and mortality during pregnancy and infancy, ${ }^{7}$ safety information on vaccination during pregnancy remains insufficient. ${ }^{8,9}$ To our knowledge, no studies focusing solely on AEFIs in pregnant women have been conducted in South Korea, and there are no published reports on AEFI surveillance systems for pregnant women. ${ }^{10}$ The AEFI studies that have been conducted in South Korea are limited by short study periods, a lack of disproportionality analyses for signal detection, and a lack of information on immunization during pregnancy. ${ }^{11,12}$

Thus, our primary objective was to evaluate the overall trends in reported AEFIs over a 13-year period and to detect signals associated with all vaccines that are available in South Korea using traditional data-mining methods to provide safety profiles for these vaccines. As an exploratory objective, we aimed to describe the number of AEFIs reported during pregnancy using the Korea Adverse Event Reporting System (KAERS) database.

\section{MATERIALS AND METHODS}

\section{Data source}

We used the Korea Institute of Drug Safety \& Risk ManagementKorea Adverse Event Reporting System Database (KIDS-KD) for January 2005 to December 2017. All spontaneous reports of AEs are accumulated in the KAERS via direct online uploading, e-mail, and telephone. These spontaneous reports are then refined by KAERS and provided as analyzable data, including data on patient demographic characteristics, general drug information, drug administration, AEs, serious AEs, type of report, type of reporter, and causality assessment. ${ }^{13}$ Serious AEs include AEs leading to hospitalization or prolonged hospitalizations, malfunction or permanent disabilities, birth defects, life-threatening illnesses, or death.

We used $\mathrm{AE}$ reports related to all vaccines from the KIDS-KD. Vaccines were coded according to the Anatomical Therapeutic Chemical classification, and AEs were coded using preferred terms (PTs) and included terms (ITs) from the WHO Adverse Reaction Terminology (WHO-ART) codes.

\section{Study vaccines}

We analyzed all vaccines reported in the KAERS, including both national immunization program (NIP) vaccines and other vaccines distributed in South Korea (Supplementary Table 1, only online), which were as follows: influenza, pneumococcal, Bacillus Calmette-Guérin (BCG), Hemophilus influenza b (Hib), human papilloma virus (HPV), meningococcal, rotavirus, diphtheria-tetanus-pertussis (DTaP, DTwP, Tdap, Td, DTap-IPV,
DTap-IPV/Hib), Japanese encephalitis, hepatitis A, hepatitis B, measles-mumps-rubella (MMR), yellow fever, polio, varicella, herpes zoster, typhoid, Hantaan virus, and cholera vaccines.

\section{Trends in overall AEFIs}

Prior to conducting analysis, the data on AEFIs were restricted to initially reported cases and suspected cases. The trends in overall AEFI reports were described for each reported year and for each vaccine. We presented vaccine-related events in a chronological sequence that included free vaccination policy on each vaccine.

To compare patterns of reported AEs for each vaccine, we used the guidelines of the Advisory Committee on Immunization Practices (ACIP) and referred to earlier studies. ${ }^{5,14}$ According to the ACIP vaccine recommendations and guidelines, AEFIs are classified as local, systemic, or allergic reactions. However, systemic reactions consist of various kinds of AEs, including neurologic reactions, which can be serious AEFIs. We, therefore, classified AEFIs into five categories: neurologic, general systemic, local, allergy, and others. These categories were all exclusive, and all AEFIs were assigned to one of the categories. Four of these categories were classified according to the WHOART System Organ Class (SOC) codes, as follows: neurologic reactions (e.g., central and peripheral nervous system disorders, autonomic nervous system disorders, and psychiatric disorders), general systemic reactions (e.g., skin and appendages disorders, musculoskeletal system disorders, collagen disorders, vision disorders, gastrointestinal system disorders, etc.), local reactions (e.g., application site disorders), and others (e.g., resistance mechanism disorders, secondary terms-events, poison specific terms). ${ }^{5}$ For allergic reactions, since there were no stipulated data listed under SOC level, we included the following allergy-related PTs: allergic reaction, allergy, anaphylaxis, anaphylactoid reaction, angioedema, hypersensitivity, and urticaria. Subsequently, we assessed and presented the frequency of the categorized AEs and the categories of AEs that were most likely to occur with a specific vaccine.

\section{Case-non-case study}

A case-non-case study is a disproportionality analysis used for signal detection or to evaluate a hypothesis in pharmacovigilance studies. ${ }^{15}$ Typically, a case-non-case study is based on twoby-two tables describing the number of AEs of interest and exposure to the drug of interest. Likewise, we used vaccine-AE pairs as the unit of analysis and defined each categorized $\mathrm{AE}$ as "cases" and the other categorized AEs as "non-cases." We assessed the reporting odds ratios (RORs) and their confidence intervals (CIs) for each categorized $\mathrm{AE}$ associated with each vaccine of interest in comparison to all other vaccines.

\section{Signal detection}

We conducted signal detections for the individual AE terms of the categorized AEs with a ROR greater than 2 from the case- 
non-case study. As signal detection is performed to identify statistically significant vaccine-AE pairs, we used causality information to analyze only those reports that were certain, probable, or possible. We performed traditional data mining using RORs. A ROR was defined as the odds of occurrence of a specific AE in a patient exposed to a drug of interest divided by the odds of occurrence of an $\mathrm{AE}$ specific to the other drug. The vaccine-AE pairs were detected as signals by meeting these indices $(\mathrm{ROR} \geq 2$, chi-square $\geq 4$, number of cases $\geq 3$ ).${ }^{16}$ After the analysis, we identified which AEs were listed on the domestic drug labels.

\section{Statistical analysis}

We analyzed all AEFIs, including serious AEs, and assessed demographics and clinical characteristics, specifically the sex and age of patients, reporting groups according to affiliations, and report types. Age was divided into seven sub-groups to assess $\mathrm{AE}$ data based on vaccination periods as follows: under 2 years, 3-6 years, 7-11 years, 12-18 years, 19-64 years, over 65 years, and unknown age. Especially for serious AEs leading to death, vaccination information and causality assessment data, as well as demographics and clinical characteristics, were evaluated. In the case-non-case study, the odds ratio and CIs of associations between each categorized $\mathrm{AE}$ and each vaccine was evaluated using logistic regression adjusted for age and sex in comparison to all other vaccines. All statistical analyses were conducted using SAS 9.4 (SAS Institute Inc., Cary, NC, USA) and Microsoft Excel (Microsoft Corp., Redmond, WA, USA). This study was approved by the institutional review board of Sungkyunkwan University (No.2019-09-008).

\section{RESULTS}

A total of 28012 AEFIs and 54378 vaccine-AE pairs were reported to KIDS-KD from 2005 to 2017. AEFI reports remained relatively consistent until 2011. The establishment of the KAERS for comprehensive management of the spontaneous $\mathrm{AE}$ reports in 2012 led to a remarkable increase in AEFI reports regardless of any type of vaccine in 2013 ( $n=7070)$; the total number of reports in 2013 was five-fold higher than that in $2011(n=1363)$. Moreover, expansion of NIP coverage for influenza vaccine during the study period also led to overall increase in AEFIs (Fig. 1).

The demographic characteristics of the patients are presented in Table 1. Females accounted for more AEFIs than males, and the frequency of reported AEFIs was relatively higher in individuals aged between 0 and 2 years. The most frequently reported vaccine was the influenza vaccine, which accounted for approximately $40 \%$ of the reports, followed by the rotavirus (11.7\%), pneumococcal (10.5\%), HPV (9.7\%), and DTaP (5.9\%) vaccines. The majority of the reported AEs were non-serious events as follows: injection site pain (23.1\%), fever (9.9\%), myalgia (7.8\%), headache (2.9\%), and vomiting (2.9\%) (Supplementary Table 2 , only online). The reported AEs were also presented in terms of SOC codes as follows: application site disorders (27.2\%), body as a whole-general disorders (18.9\%), respiratory system disorders $(12.8 \%)$, gastrointestinal system disorders (8.1\%), and central and peripheral nervous system disorders (7.7\%) (Supplementary Table 3, only online).

Categorized AEs are described in Fig. 2. Among AEFIs for vaccines, general systemic reactions were most common, followed by local reactions and neurologic reactions. Neurologic reactions

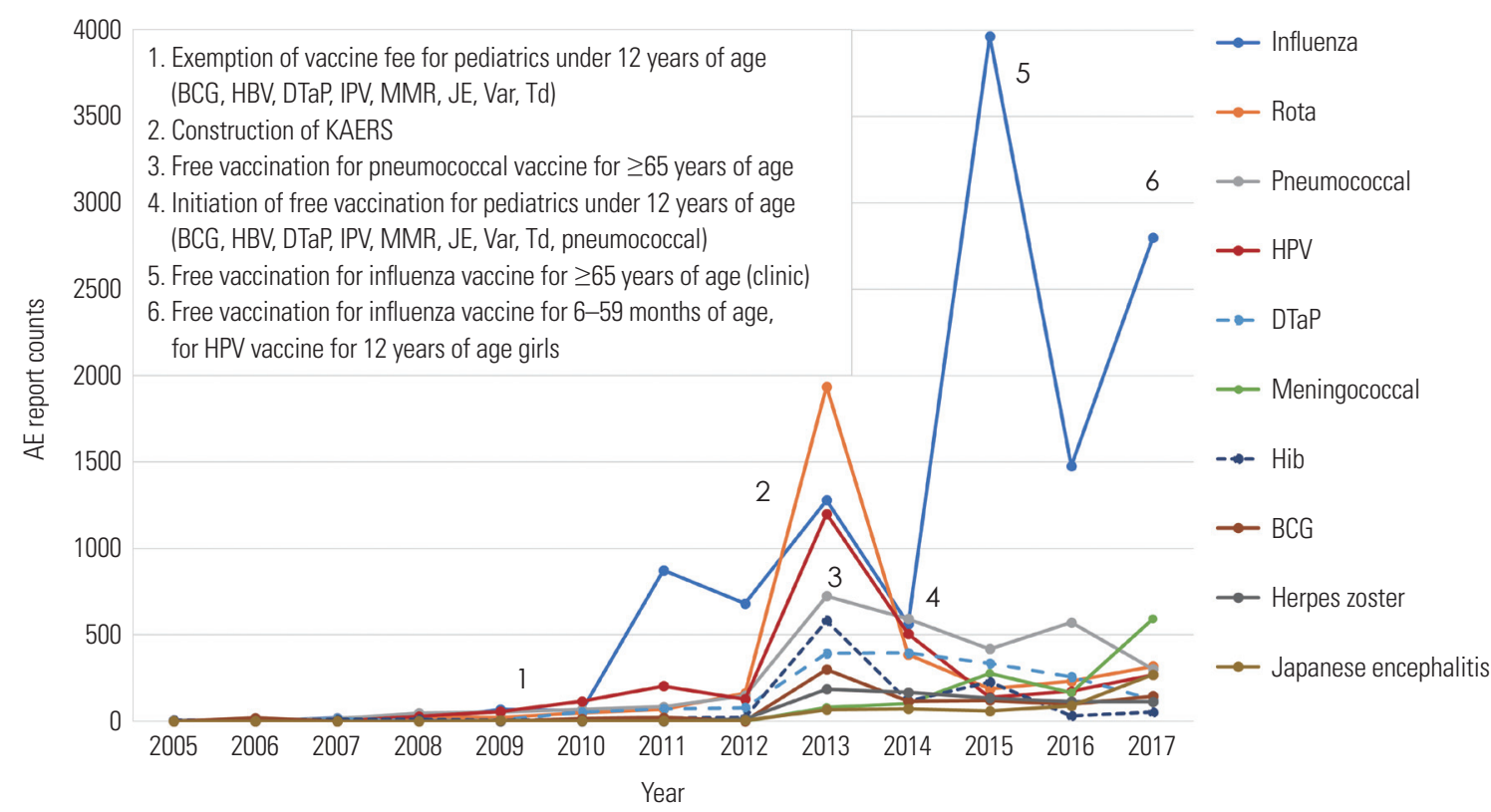

Fig. 1. Trends in the numbers of AEs following immunization according to the 10 most reported vaccines in the KAERS from 2005 to 2017 . $A E$, adverse event; KAERS, Korea Adverse Event Reporting System; BCG, Bacillus Calmette-Guérin; HBV, Hepatitis B virus; DTaP, diphtheria, tetanus, acellular pertussis; IPV, inactivated polio virus; MMR, measles mumps rubella; JE, Japanese encephalitis; Var, Varicella zoster; Td, Tetanus-diphtheria toxoid; HPV, human papilloma virus; Hib, hemophilus influenza type $b$. 
were reported in high frequency following vaccinations with the rotavirus vaccine, meningococcal vaccine, and yellow fever vaccine. General systemic reactions accounted for the largest proportion of AEs; they were reported most frequently in association with the BCG vaccine. Local reactions were reported in high frequency in association with pneumococcal vaccine, meningococcal vaccine, and herpes zoster vaccine. However, local reactions were barely reported in association with the rotavirus vaccine because it is administered orally. Allergic reactions were reported in association with the rotavirus, Hib, Japanese encephalitis, varicella, and MMR vaccines. The vaccine-AE pairs

Table 1. Demographics and Characteristics of AEs Following Immunization from 2005 to 2017

\begin{tabular}{|c|c|c|}
\hline \multirow{2}{*}{ Demographics and characteristics } & \multicolumn{2}{|c|}{ AE report (serious AEs) } \\
\hline & $\mathbf{n}$ & $\%$ \\
\hline \multicolumn{3}{|l|}{ Sex } \\
\hline Male & $9580(762)$ & $34.2(39.1)$ \\
\hline Female & $17332(1020)$ & $61.9(52.3)$ \\
\hline Unknown & $1100(167)$ & $3.9(8.6)$ \\
\hline \multicolumn{3}{|l|}{ Age (yr) } \\
\hline $0-2$ & $4291(312)$ & $15.3(16.0)$ \\
\hline $3-6$ & $1407(167)$ & $5.0(8.6)$ \\
\hline $7-11$ & $1187(150)$ & $4.2(7.7)$ \\
\hline $12-18$ & $1586(285)$ & $5.7(14.6)$ \\
\hline $19-64$ & $9250(515)$ & $33.0(26.4)$ \\
\hline$\geq 65$ & $1597(191)$ & $5.7(9.8)$ \\
\hline Unknown & 8694 (329) & $31.0(16.9)$ \\
\hline \multicolumn{3}{|l|}{ Serious AEs } \\
\hline $\begin{array}{l}\text { Hospitalization or prolongation of } \\
\text { hospitalization }\end{array}$ & 1296 & 4.6 \\
\hline Disability & 24 & 0.1 \\
\hline Birth defect & - & - \\
\hline Life threatening & 21 & 0.1 \\
\hline Death & 107 & 0.4 \\
\hline Other & 629 & 2.2 \\
\hline \multicolumn{3}{|l|}{ Report type } \\
\hline Spontaneous report & $13157(1180)$ & $47.0(60.5)$ \\
\hline Postmarketing surveillance report* & 14501 (697) & $51.8(35.8)$ \\
\hline Literature & $50(28)$ & $0.2(1.4)$ \\
\hline Other & $304(44)$ & $1.1(2.3)$ \\
\hline \multicolumn{3}{|l|}{ Reporting groups by affiliation } \\
\hline Regional pharmacovigilance center & $5596(113)$ & $20.0(5.8)$ \\
\hline Manufacturer & $19936(1563)$ & $71.2(80.2)$ \\
\hline Medical institution & $350(6)$ & $1.2(0.3)$ \\
\hline Pharmacy & $4(0)$ & $0.0(0.0)$ \\
\hline Public health center & $62(2)$ & $0.2(0.1)$ \\
\hline Consumer & $416(51)$ & $1.5(2.6)$ \\
\hline Other & $1648(214)$ & $5.9(11.0)$ \\
\hline Total & 28012 (1949) & $100(100)$ \\
\hline
\end{tabular}

$\mathrm{AE}$, adverse event.

*Postmarketing surveillance reports are required for drug approval by the Korea Ministry of Food and Drug Safety. are presented in Supplementary Table 4 (only online).

In the case-non-case study, there were notable relations between vaccines and the categorized AEs as follows: rotavirus vaccine-neurologic reactions, meningococcal vaccine-local reactions, BCG vaccine-general systemic reactions and local reactions, and Japanese encephalitis vaccine-allergic reactions (Table 2). For these pairs, we performed conventional signal detection analysis, which met the data-mining index of ROR. As a result, nervousness was seen to be related with the rotavirus vaccine, and an injection site mass was found to be related with the meningococcal vaccine. For the BCG vaccine, lymphadenitis was particularly reported at high frequency with great disproportionality (ROR, 804.48; 95\% CI, 710.42-911.00). Additionally, injection site infections and injection site abscess sterile were frequent following BCG vaccination. Also, acne was observed as a new signal, which is not listed on the label of the BCG vaccine. Allergic reactions were commonly observed for the Japanese encephalitis vaccine; these reactions included hypersensitivity, urticaria, anaphylactoid reactions, and allergy as signals (Table 3 ).

A total of 1949 serious AEs were reported, and our results revealed that 107 serious AEFIs resulted in death. Of the 107 reported deaths, a higher number occurred in males $(n=56)$ than in females $(n=39)$, children under the age of 7 years $(n=55)$, and older adults above 65 years of age $(n=26)$. The majority of vaccines were influenza $(n=35), D T a P(n=31)$, and IPV $(n=22)$ vaccines (Table 4).

\section{DISCUSSION}

In this study, we described the overall trends in reported AEFIs among the general population including pregnant women over a 13-year period using the KAERS database. The frequency of spontaneous AE reports followed an increasing trend. This increase was attributed to development of the surveillance system and the expansion of free vaccination coverage. For example, regarding the influenza vaccine, one of the most widely used vaccines in South Korea, the coverage of free vaccination has significantly expanded. ${ }^{17}$ In line with this increased coverage, the influenza vaccine accounted for approximately $40 \%$ of reports in the KAERS database. Research on AEFI surveillance has been conducted in several countries. Our findings show similar AE reporting patterns to preceding surveillance studies in the U.S. and Australia who use spontaneous AE reporting system databases. However, regarding the BCG vaccine, which is not routinely recommend for the general population in the US or Australia, the number of reported AEFIs for the BCG vaccine showed a significant difference between South Korea and other nations. $^{3,4}$

Among a total of 54378 AEs, most AEs were stipulated in the Vaccine Information Statements of Centers for Disease Control and Prevention, US, ${ }^{18}$ although certain serious AEFIs should be 
monitored. In our study, among 1949 cases of serious AEs, 107 serious AEFIs resulted in death. However, these results should be interpreted with caution for the following reasons: First, in the majority of cases, causal assessment information was unknown or unclassified. Second, as vaccination is strongly recommended by the NIP in children and older adults, a high frequency of deaths was observed in these populations. Lastly, influenza and DTaP vaccines are widely used not only in children and older adults, but also in pregnant women. For this reason, the numbers of influenza and DTaP vaccines were higher than those for other vaccines, and further research is needed to establish the safety profile of these vaccines.

The rotavirus vaccine showed an association with neurologic reactions, most of which were vomiting and diarrhea. Among

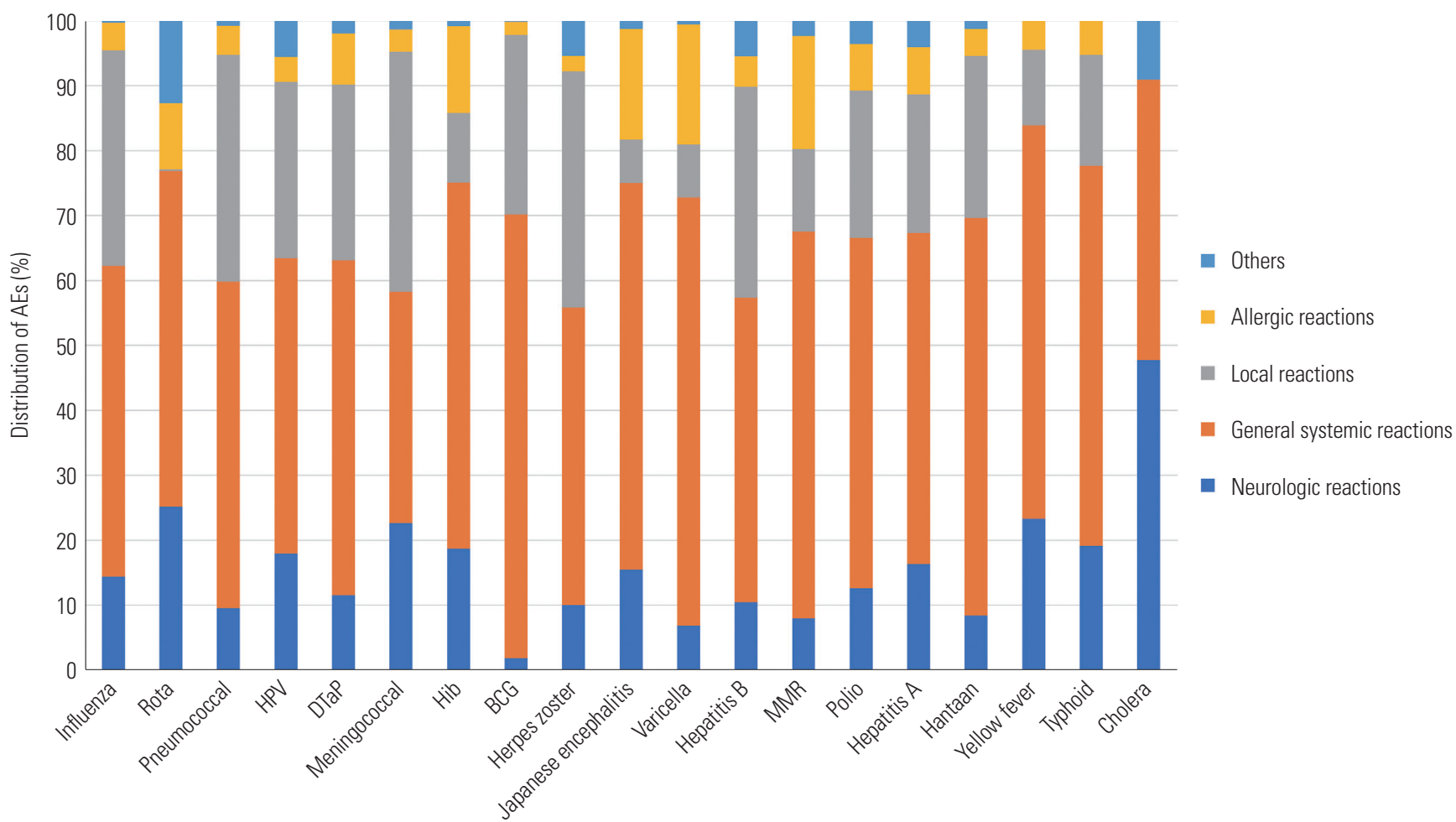

Fig. 2. Distribution of the four categorized AEs following immunization by vaccines reported in the KAERS from 2005 to 2017. AE, adverse event; KAERS, Korea Adverse Event Reporting System; HPV, human papilloma virus; DTaP, diphtheria, tetanus, acellular pertussis; Hib, hemophilus influenza type b; BCG, Bacillus Calmette-Guérin; MMR, measles mumps rubella.

Table 2. aROR of Case-Non-Case Studies on the Basis of Categorized Adverse Events Following Immunization for the Top 10 Reported Vaccines

\begin{tabular}{|c|c|c|c|c|c|c|c|c|}
\hline \multirow{2}{*}{ Vaccines } & \multicolumn{2}{|c|}{ Neurologic reaction* } & \multicolumn{2}{|c|}{ General systemic reaction } & \multicolumn{2}{|c|}{ Local reaction $^{\ddagger}$} & \multicolumn{2}{|c|}{ Allergic reaction } \\
\hline & $\mathrm{AROR}^{1}$ & $95 \% \mathrm{Cl}$ & aROR & $95 \% \mathrm{Cl}$ & aROR & $95 \%$ CI & aROR & $95 \% \mathrm{CI}$ \\
\hline Influenza & 0.88 & $0.83-0.93$ & 0.95 & $0.91-0.99$ & 1.32 & $1.27-1.38$ & 0.85 & $0.77-0.92$ \\
\hline Rotavirus & 2.43 & $2.25-2.62$ & 0.96 & $0.91-1.02$ & 0.01 & $0.01-0.01$ & 1.46 & $1.31-1.62$ \\
\hline Pneumococcal & 0.62 & $0.57-0.68$ & 1.04 & $0.99-1.10$ & 1.45 & $1.37-1.53$ & 0.77 & $0.68-0.87$ \\
\hline HPV & 1.45 & $1.34-1.57$ & 0.90 & $0.85-0.96$ & 0.75 & $0.70-0.80$ & 0.83 & $0.71-0.97$ \\
\hline DTaP & 0.66 & $0.59-0.74$ & 1.07 & $0.99-1.16$ & 1.29 & $1.18-1.41$ & 1.08 & $0.93-1.24$ \\
\hline Meningococcal & 1.41 & $1.27-1.56$ & 0.52 & $0.48-0.56$ & 2.88 & $2.63-3.16$ & 0.37 & $0.30-0.46$ \\
\hline Hib & 0.96 & $0.86-1.08$ & 1.29 & $1.18-1.41$ & 0.66 & $0.58-0.76$ & 1.78 & $1.55-2.03$ \\
\hline BCG & 0.07 & $0.04-0.11$ & 2.20 & $1.91-2.53$ & 3.15 & $2.69-3.68$ & 0.17 & $0.11-0.27$ \\
\hline Herpes zoster & 0.73 & $0.61-0.87$ & 0.97 & $0.87-1.08$ & 1.13 & $1.01-1.26$ & 0.63 & $0.44-0.89$ \\
\hline Japanese encephalitis & 0.87 & $0.72-1.05$ & 1.36 & $1.18-1.56$ & 0.29 & $0.22-0.38$ & 2.38 & $1.98-2.87$ \\
\hline
\end{tabular}

BCG, Bacillus-Calmette-Guérin; Hib, hemophilus influenzae type b; HPV, human papilloma virus; DTaP, diphtheria tetanus acellular pertussis; Cl, confidence interval; aROR, adjusted reporting odds ratios; WHO-ART SOC, WHO-Adverse Reaction Terminology System Organ Class; PT, preferred term.

*Neurologic reactions include the following WHO-ART SOC codes: central and peripheral nervous system disorders, autonomic nervous system disorders, and psychiatric disorders, ${ }^{\dagger}$ General systemic reactions include the following WHO-ART SOC codes: skin and appendages disorders, musculoskeletal system disorders, collagen disorders, vision disorders, gastrointestinal system disorders, etc, "Local reactions include the following WHO-ART SOC codes: application site disorders, ${ }^{\text {s} A l l e r g i c ~ r e a c t i o n s ~ i n c l u d e ~ t h e ~ f o l l o w i n g ~ W H O-A R T ~ P T ~ c o d e s: ~ g e n e r a l i z e d ~ u r t i c a r i a, ~ a n a p h y l a c t i c ~ r e a c t i o n s, ~ a l l e r g y, ~ h y p e r s e n s i t i v i t y, ~ e t c, ~ " A d j u s t e d ~ f o r ~}$ age and sex. 
Table 3. Detected Signals among Categorized AEs of Vaccines that Meet the Data-Mining Index in the Case-Non-Case Study

\begin{tabular}{|c|c|c|c|c|c|}
\hline AE category* & AE & n & ROR & $95 \% \mathrm{Cl}$ & Drug label $^{\dagger}$ \\
\hline \multicolumn{6}{|l|}{ Rotavirus } \\
\hline Neurologic reaction & Nervousness & 21 & 4.31 & $2.81-6.62$ & Y \\
\hline \multicolumn{6}{|l|}{ Meningococcal } \\
\hline Local reaction & Injection site mass & 39 & 11.51 & 8.76-15.14 & Y \\
\hline \multicolumn{6}{|l|}{ BCG } \\
\hline \multirow[t]{2}{*}{ General systemic reaction } & Lymphadenitis & 363 & 804.48 & 710.42-911.00 & Y \\
\hline & Acne & 3 & 13.99 & $5.41-36.17$ & N \\
\hline \multirow[t]{2}{*}{ Local reaction } & Injection site infection & 124 & 57.56 & 49.92-66.37 & Y \\
\hline & Injection site abscess sterile & 27 & 130.98 & 108.48-158.16 & Y \\
\hline \multicolumn{6}{|l|}{ Japanese encephalitis } \\
\hline \multirow[t]{4}{*}{ Allergic reaction } & Hypersensitivity & 31 & 8.56 & 6.04-12.12 & Y \\
\hline & Urticaria & 11 & 4.34 & $2.43-7.76$ & Y \\
\hline & Anaphylactoid reaction & 3 & 13.62 & 4.89-37.97 & Y \\
\hline & Allergy & 3 & 7.70 & $2.64-22.42$ & Y \\
\hline
\end{tabular}

$\mathrm{AE}$, adverse event; $\mathrm{BCG}$, Bacillus-Calmette-Guérin; ROR, reporting odds ratio; $\mathrm{Cl}$, confidence interval; $\mathrm{Y}$, yes; N, no.

${ }^{*}$ Categorized adverse events with $\mathrm{RROR} \geq 2$ in the case/non-case approach were included in the signal detection analysis, ${ }^{\dagger}$ Indicting whether AEs were listed on the domestic drug label.

neurologic reactions, nervousness was detected as a signal. Although the AEs were transient and non-serious, patients should be informed regarding potential AEs at the time of vaccination.

Most of the BCG vaccine-related AE reports were for general systemic reactions and local reactions, which were mostly lymphadenitis and injection site infections, respectively; both these AEs were detected as signals. Lymphadenitis, defined as the development of regional lymph node enlargement, is the most common complication of the BCG vaccine. The risk of BCG-related lymphadenitis has been explored previously in South Korea, with the incidence rate of lymphadenitis ranging from $0.20 \%$ to $0.69 \% .{ }^{19}$ Despite the incidence rate being generally high in the context of vaccine-induced AEs, most of these complications are self-resolving. In fact, numerous studies have discussed the management of lymphadenitis, with one study conducted in the UK reporting that $78 \%$ of all cases were managed conservatively and self-resolved within 6 months. ${ }^{20}$ Additionally, the South Korean government operates a routine compensation system for AEs and has been providing compensation for $95 \%$ of the claims regarding BCG. ${ }^{21,22}$

In our study, the Japanese encephalitis vaccine showed an association with allergic reactions, most of which were hypersensitivity reactions and urticaria. Allergic reactions to the Japanese encephalitis vaccine, including urticaria, anaphylaxis, and angioedema, have been reported in several studies. ${ }^{23,24}$ Urticaria is a common and well-recognized complication of the Japanese encephalitis vaccine, but anaphylaxis and angioedema may be serious. In this study, a few cases of anaphylactic reaction and angioedema were observed in the population immunized for Japanese encephalitis. This may be caused by the immunogens or excipients of the vaccine, ${ }^{25}$ although the exact cause remains to be identified.

For our exploratory objective, we described all AEFIs during pregnancy. We screened the KIDS-KD for patients who were listed under the WHO-ART PT 2221 code "exposure during pregnancy" and classified them as pregnant women. ${ }^{8}$ The number of AEFIs reported during pregnancy was extremely low, with only 61 vaccine-AE pairs. Five vaccines were reported, as follows: HPV ( $n=33)$, DTaP $(n=15)$, herpes zoster $(n=10)$, hepatitis $B(n=2)$, and MMR $(n=1)$. Most AEs were non-serious, but abortions $(n=7)$ were reported only for the HPV vaccine (Table 5). While this suggests that an association between abortions and HPV vaccines exists, no association was found between HPV vaccinations and spontaneous abortions in a recent nationwide observational study. ${ }^{26}$ The current immunization guidelines for pregnant women recommend inactivated influenza and Tdap vaccines. ${ }^{27}$ However, in our study, AEFIs during pregnancy were reported for HPV, herpes zoster, hepatitis B, and MMR vaccines. Considering these reported vaccines, pregnant women may have been vaccinated before knowing that they were pregnant. Further research is needed to confirm vaccine safety and associations between vaccines and AEs and development, as optimized vaccination regimens in pregnancy are important to maternal and fetal health.

To promote public health, free vaccination coverage has expanded during recent decades in South Korea. In 2009, the Korea Centers for Disease Control \& Prevention (KCDC) implemented an immunization policy that exempted vaccine fees for pediatric patients under 12 years of age for eight types of vaccines (BCG, hepatitis B, DTaP, inactivated polio, MMR, Japanese encephalitis, varicella, and Td vaccines). Following the introduction of an immunization policy for these eight vaccines in 2009, vaccination coverage has gradually expanded, and as of 2019, 17 types of vaccines are administered free of charge.$^{28}$ For the influenza vaccine, which is widely used in South Korea, free vaccination coverage has been extended from the pediatric 
Table 4. Demographics and Characteristics of Serious AEs Following Immunization Leading to Death from 2005 to 2017

\begin{tabular}{|c|c|}
\hline Demographics and characteristics & AE report, $n(\%)$ \\
\hline \multicolumn{2}{|l|}{ Sex } \\
\hline Male & $56(52.3)$ \\
\hline Female & $39(36.5)$ \\
\hline Unknown & $12(11.2)$ \\
\hline \multicolumn{2}{|l|}{ Age (yr) } \\
\hline $0-2$ & $34(31.8)$ \\
\hline $3-6$ & $21(19.6)$ \\
\hline $7-11$ & $3(2.8)$ \\
\hline $12-18$ & $4(3.7)$ \\
\hline $19-64$ & $9(8.4)$ \\
\hline$\geq 65$ & $26(24.3)$ \\
\hline Unknown & $10(9.4)$ \\
\hline \multicolumn{2}{|l|}{ Type of vaccine* } \\
\hline Influenza & $35(25.7)$ \\
\hline DTaP & $31(22.8)$ \\
\hline IPV & $22(16.2)$ \\
\hline Pneumococcal & $13(9.6)$ \\
\hline Hib & $12(8.8)$ \\
\hline Hepatitis B & $12(8.8)$ \\
\hline Rotavirus & $6(4.4)$ \\
\hline BCG & $2(1.5)$ \\
\hline MMR & $1(0.7)$ \\
\hline Japanese encephalitis & $1(0.7)$ \\
\hline Varicella & $1(0.7)$ \\
\hline \multicolumn{2}{|l|}{ Causal assessment information } \\
\hline Certain & $0(0)$ \\
\hline Probable & $3(0.03)$ \\
\hline Possible & $6(0.06)$ \\
\hline Unlikely & $5(0.05)$ \\
\hline Unclassified & $26(24.3)$ \\
\hline Not applicable & $0(0)$ \\
\hline Unknown & 67 (62.6) \\
\hline Total & 107 (100) \\
\hline
\end{tabular}

AE, adverse event; BCG, Bacillus Calmette-Guérin; Hib, hemophilus influenza type b; DTaP, diphtheria, tetanus, acellular pertussis; MMR, measles, mumps, rubella; IPV, inactivated polio virus.

${ }^{*}$ As a recipient could be vaccinated with more than one vaccine, the total number exceeds 107.

population under 12 years of age to older adults 65 years of age and older, and in 2019, pregnant women have also been included. The KCDC has been expanding the immunization coverage, although the risk-benefit evaluation of vaccination and continuous AEFI surveillance are imperative for future healthcare policies.

The strengths of this study were that the trends of reported AEFIs associated with vaccines approved in South Korea were comprehensively analyzed, including signal detection and evaluation of AEFIs in pregnancy. Our study findings can provide a fundamental basis for safe immunization in the future. How-
Table 5. AEs Following Immunization during Pregnancy Reported in KAERS from 2005 to 2017

\begin{tabular}{lc}
\hline \multicolumn{1}{c}{ Vaccines } & AEs, $\mathbf{n}(\%)$ \\
\hline HPV & $33(54.1)$ \\
\hline Inappropriate schedule of drug administration & $16(26.2)$ \\
\hline Abortion & $7(11.5)$ \\
\hline Injection site reaction & $4(6.6)$ \\
\hline $\begin{array}{l}\text { Cervical dysplasia, medication error, pyrexia, vaccine } \\
\text { failure, chills, unintended pregnancy* }\end{array}$ & $6(9.8)$ \\
DTaP & $15(24.6)$ \\
\hline Injection site reaction & $4(6.6)$ \\
\hline Unintended pregnancy & $2(3.3)$ \\
\hline $\begin{array}{l}\text { Pruritus, muscle stiffness, myalgia, rash, pain in limb, } \\
\text { pigmentation abnormal, blisters, paresthesia distal, skin } \\
\text { laceration* }\end{array}$ & $9(14.8)$ \\
Herpes Zoster & $10(16.4)$ \\
\hline Inappropriate schedule of drug administration & $5(8.2)$ \\
\hline Medication error & $2(3.3)$ \\
\hline $\begin{array}{l}\text { Small for gestational age, incorrect drug administration } \\
\text { route, rhinitis* }\end{array}$ & $3(4.9)$ \\
\hline Hepatitis B & $2(3.3)$ \\
\hline Inappropriate schedule of drug administration, off label use & $2(3.3)$ \\
MMR & $1(1.6)$ \\
\hline Medication error & $1(1.6)$ \\
\hline Total & $61(100)$ \\
\hline AE, & \\
\hline
\end{tabular}

$\mathrm{AE}$, adverse event; KAERS, Korea Adverse Event Reporting System; HPV, human papilloma virus; DTaP, diphtheria tetanus acellular pertussis; MMR, measles mumps rubella.

*Each adverse event was reported in only one case.

ever, some limitations remain in our study. First, due to the inherent limitations of a spontaneous reporting database, inconsistent quality of data and underestimation of AE reporting are possible. The degree of underestimation may have been substantial, especially from 2005 to 2012 , as the spontaneous AE reporting system was not yet operational during this period. Nevertheless, the AE reporting volume increased significantly upon the introduction of the KIDS-KAERS in 2013, and our study analyzed all vaccine-related AEs reported up until 2017. Notwithstanding the inherent limitation of potential underreporting, our findings provide insights into the differential safety profiles of all NIP vaccines, which previously has not been established in South Korea. Second, calculating incidence rates of AEFIs is limited by the lack of denominator data. Third, potential misclassifications of AEs into the five specified categories may have occurred. Last, while defining pregnant women in the database, underestimation of the number of pregnant women is possible.

In conclusion, there are several factors that affect AEFIs, such as the status of the vaccinated person, type of vaccines, immunogenicity of vaccines, and excipients in the vaccines. Our findings show that the safety profiles of vaccines were similar to those in other nations, except for the BCG vaccine. Most AEFIs were non-serious and were general systemic reactions. A com- 
prehensive AEFI monitoring system and continuous vaccine surveillance need to be undertaken. With sufficient knowledge of AEFIs, providing adequate safety information to both healthcare providers and patients may help improve vaccination rates. Ultimately, a proactive response to current public health threats from vaccine-preventable diseases will be needed.

\section{ACKNOWLEDGEMENTS}

This study was supported by the Health Fellowship Foundation and Government-wide R\&D Fund project for infectious disease research (grant number: HG18C0068).

\section{AUTHOR CONTRIBUTIONS}

Conceptualization: Dongwon Yoon and Ju-Young Shin. Data curation: Dongwon Yoon and Hyesung Lee. Formal analysis: Dongwon Yoon. Funding acquisition: Ju-Young Shin. Investigation: Dongwon Yoon. Methodology: Dongwon Yoon and Hyesung Lee. Project administration: Ju-Young Shin. Resources: Hyesung Lee. Software: Dongwon Yoon and Hyesung Lee. Supervision: Ju-Young Shin. Validation: Ju Hwan Kim and Hyesung Lee. Visualization: Dongwon Yoon. Writing-original draft: Dongwon Yoon. Writing_-review \& editing: Ju Hwan Kim and Ju-Young Shin. Approval of final manuscript: all authors.

\section{ORCID iDs}

Dongwon Yoon

Ju Hwan Kim Hyesung Lee https://orcid.org/0000-0002-9369-0789 Ju-Young Shin https://orcid.org/0000-0001-7253-6515 https://orcid.org/0000-0001-6556-9984 https://orcid.org/0000-0003-1010-7525

\section{REFERENCES}

1. World Health Organization. Ten threats to global health in 2019 [accessd on 2019 August 13]. Available at: https://www.who.int/ emergencies/ten-threats-to-global-health-in-2019.

2. Dubé E, Laberge C, Guay M, Bramadat P, Roy R, Bettinger J. Vaccine hesitancy: an overview. Hum Vaccin Immunother 2013;9: 1763-73.

3. Zhou W, Pool V, Iskander JK, English-Bullard R, Ball R, Wise RP, et al. Surveillance for safety after immunization: Vaccine Adverse Event Reporting System (VAERS)--United States, 1991-2001. MMWR Surveill Summ 2003;52:1-24.

4. Dey A, Wang H, Quinn H, Hiam R, Wood N, Beard F, et al. Surveillance of adverse events following immunisation in Australia annual report, 2017. Commun Dis Intell (2018) 2019;43.

5. Schumacher Z, Bourquin C, Heininger U. Surveillance for adverse events following immunization (AEFI) in Switzerland--1991-2001. Vaccine 2010;28:4059-64.

6. Sato APS, Ferreira VLR, Tauil MC, Rodrigues LC, Barros MB, Martineli E, et al. Use of electronic immunization registry in the surveillance of adverse events following immunization. Rev Saude Publica 2018;52:4.

7. Rasmussen SA, Watson AK, Kennedy ED, Broder KR, Jamieson DJ. Vaccines and pregnancy: past, present, and future. Semin Fetal Neonatal Med 2014;19:161-9.

8. Moro PL, Cragan J, Lewis P, Sukumaran L. Major Birth Defects after Vaccination Reported to the Vaccine Adverse Event Reporting System (VAERS), 1990 to 2014. Birth Defects Res 2017;109:1057-62.
9. Fulton TR, Narayanan D, Bonhoeffer J, Ortiz JR, Lambach P, Omer SB. A systematic review of adverse events following immunization during pregnancy and the newborn period. Vaccine 2015;33: 6453-65.

10. Cassidy C, MacDonald NE, Steenbeek A, Top KA. Adverse event following immunization surveillance systems for pregnant women and their infants: a systematic review. Pharmacoepidemiol Drug Saf 2015;24:361-7.

11. Choe YJ, Bae GR. Management of vaccine safety in Korea. Clin Exp Vaccine Res 2013;2:40-5.

12. Cha HG. Analysis of adverse events following immunization using The Korea Adverse Event Reporting System. J Welln 2016;11:459-70.

13. Korea Institute of Drug Safety and Risk Management. Guideline for use of Korea Adverse Event Reporting System Database, 2019 [accessed on 2020 March 17]. Available at: https://open.drugsafe. or.kr/original/guidelines/Read.jsp?ntt_id=2171.

14. Centers for Disease Control and Prevention. Vaccine Recommendations and Guidelines of the ACIP, 2019 [accessed on 2019 June 17]. Available at: https://www.cdc.gov/vaccines/hcp/acip-recs/ general-recs/adverse-reactions.html.

15. Faillie JL. Case-non-case studies: principle, methods, bias and interpretation. Therapie 2019;74:225-32.

16. Wilson AM, Thabane L, Holbrook A. Application of data mining techniques in pharmacovigilance. Br J Clin Pharmacol 2004;57:127-34.

17. Yun JW, Noh JY, Song JY, Chun C, Kim Y, Cheong HJ. The Korean influenza national immunization program: history and present status. Infect Chemother 2017;49:247-54.

18. Centers for Disease Control and Prevention (CDC). Vaccine Information Statements, 2019 [accessed on 2019 November 12]. Available at: https://www.cdc.gov/vaccines/hcp/vis/index.html.

19. Kim J, Lee K, Kim JH, Kim SJ, Lee SY, Lee HJ, et al. The incidence rate of lymphadenitis after Bacille Calmette-Guérin (BCG) vaccination. Pediatr Infect Vaccine 2016;23:54-61.

20. Venkataraman A, Yusuff M, Liebeschuetz S, Riddell A, Prendergast AJ. Management and outcome of Bacille Calmette-Guérin vaccine adverse reactions. Vaccine 2015;33:5470-4.

21. An SW, Noh Y, Baek YH, Park HH, Park SH, Park S, et al. Current status of adverse drug reaction relief system in Korea, Japan and Taiwan. Yakhak Hoeji 2019;63:214-20.

22. Kim MK, Lee YK, Kim TE, Kong I, Yang HJ, Suh ES. Surveillance and compensation claims for adverse events following immunization from 2011 to 2016 in the Republic of Korea. Clin Exp Vaccine Res 2017;6:146-55.

23. Plesner AM. Allergic reactions to Japanese encephalitis vaccine. Immunol Allergy Clin North Am 2003;23:665-97.

24. Walker WL, Hills SL, Miller ER, Fischer M, Rabe IB. Adverse events following vaccination with an inactivated, Vero cell culture-derived Japanese encephalitis vaccine in the United States, 20122016. Vaccine 2018;36:4369-74.

25. McCallum AD, Jones ME. Allergy to IXIARO and BIKEN Japanese encephalitis vaccines. J Travel Med 2013;20:60-2.

26. Scheller NM, Pasternak B, Mølgaard-Nielsen D, Svanström H, Hviid A. Quadrivalent HPV vaccination and the risk of adverse pregnancy outcomes. N Engl J Med 2017;376:1223-33.

27. Advisory Committee on Immunization Practices. Guiding Principles for Development of ACIP Recommendations for Vaccination during Pregnancy and Breastfeeding, 2008 [accessed on 2019 November 12]. Available at: https://www.cdc.gov/vaccines/hcp/ acip-recs/rec-vac-preg.html?CDC_AA_refVal=https\%3A\%2F\%2 Fwww.cdc.gov\%2Fvaccines\%2Facip\%2Fcommittee\%2Fguidance \%2Frec-vac-preg.html.

28. Korea Centers for Disease Control \& Prevention. National Immunization Program for pediatrics in Korea, 2019 [accessed on 2019 October 4]. Available at: https://nip.cdc.go.kr/irgd/introduce. do?MnLv1=3\&MnLv2=1\&MnLv3=1. 